\title{
Provision of critical care in austere conditions: staff, supplies and space
}

\author{
Jasmine Proulx ${ }^{1}$, Katherine Russell ${ }^{2}$, Julien Gallant ${ }^{2}$ and Kristina Krmpotic ${ }^{1,2,3^{*}}$ (B)
}

(C) 2021 Springer-Verlag GmbH Germany, part of Springer Nature

In a recent issue of Intensive Care Medicine, Proudfoot et al. [1] describe a cohort of invasively ventilated patients with coronavirus disease 2019 (COVID-19) cared for at Nightingale Hospital London (NHL), a critical care unit constructed in a London conference centre. It is commendable that outcomes were comparable to a national cohort of patients with similar characteristics and severity of illness. Provision of critical care requires significant infrastructure, including specialized personnel and equipment [2], which were rapidly mobilized to provide exceptional care.

The authors note two key logistical challenges, including no predefined organizational structure or staffing model and lack of existing oxygen supply. We similarly identified staffing, equipment, and space as common themes mapped in our recently conducted unpublished scoping review surrounding the provision of critical care in austere environments during non-pandemic civilian disaster situations. Our search for and screening of English publications indexed from 1990 to April 82020 yielded 14 publications describing actual or theoretical field hospitals and mobile units with critical care capacity in developed countries (Supplementary materials). Publications acknowledged critical care healthcare providers as the most valuable resource, and emphasized the importance of adhering to intensivist-led models and having predefined plans in place for organizational structure. Resource considerations included strategies to optimize oxygen supply, and pharmaceuticals needed to manage chronic diseases. A significant proportion of patients require treatment for population health

\footnotetext{
*Correspondence: kristina.krmpotic@iwk.nshealth.ca

2 Department of Pediatric Critical Care, IWK Health, 5850/5980 University Ave, Box 9700, Halifax, NS B3K 6R8, Canada
}

Full author information is available at the end of the article conditions. Capacity for respiratory isolation and climate control were highlighted. Equipoise was noted between adherence to the detail-oriented quality routines of established critical care units and inevitable modifications. No studies of actual events reported the need to make triage decisions related to limitations of therapy, but no scenarios were as devastating as the COVID-19 pandemic. Proudfoot et al. do not describe whether NHL adhered to routine protocols, or if compromises were (understandably) necessary with the significant strain on resources.

When local resources are overwhelmed following a natural disaster or mass casualty event, preparedness for evacuation and patient diversion may be adequate [3] Local epidemics may be supported sufficiently by surge capacity planning for staff, supplies, and space, with contingency response (100\% above baseline) similarly relying on regional collaboration [3, 4]. However, the COVID-19 pandemic has taught us that these strategies are insufficient when healthcare infrastructure is compromised on a wider scale. Surge crisis response (200\% above baseline) requires mobilization of national and sometimes international aid [3, 4]. The worldwide nature of this disaster has resulted in an inability to access these resources. Even in well-prepared areas, maximal surge capacity has been exceeded due to high case rates and the prolonged nature of the pandemic. This has resulted in provision of critical care under austere conditions, including field hospitals staffed by non-critical care trained healthcare providers, and resource allocation necessitating reverse triage. There is much to be learned from studying the rapid mobilization of resources and provision of critical care in austere environments such as NHL and those identified by our review. In the future, we can be better prepared for global pandemic response. 


\section{Supplementary Information}

The online version contains supplementary material available at https://doi, org/10.1007/s00134-021-06456-5.

\section{Author details}

${ }^{1}$ Faculty of Medicine, Dalhousie University, Halifax, Canada. ${ }^{2}$ Department of Pediatric Critical Care, IWK Health, 5850/5980 University Ave, Box 9700, Halifax, NS B3K 6R8, Canada. ${ }^{3}$ Department of Critical Care, Dalhousie University, Halifax, Canada.

\section{Acknowledgements}

We thank librarian Jackie Phinney for assistance in developing the search strategy and reviewing the manuscript.

\section{Author contributions}

JP and KK designed the study. JP developed the search strategy. JP, KR, and JG screened articles and extracted data. All authors contributed to the interpretation of data. JP and KK drafted the manuscript. All authors critically reviewed the manuscript and approved the final version.

\section{Funding}

No specific funding was provided for this study.

\section{Declarations}

\section{Conflicts of interest}

The authors declare that they have no conflicts of interest.

\section{Ethics approval}

This study did not require ethics approval.

\section{Publisher's Note}

Springer Nature remains neutral with regard to jurisdictional claims in published maps and institutional affiliations.

Accepted: 7 June 2021

Published online: 18 June 2021

\section{References}

1. Proudfoot AG, O'Brien B, Schilling R, Gould DW, McGlennan A et al (2021) Rapid establishment of a COVID-19 critical care unit in a convention Centre: the Nightingale Hospital London experience. Intensive Care Med 47:349-351. https://doi.org/10.1007/s00134-020-06334-6

2. Marshall JC, Bosco L, Adhikari NK et al (2017) What is an intensive care unit? A report of the task force of the World Federation of Societies of Intensive and Critical Care Medicine. J Crit Care 37:270-276. https://doi. org/10.1016/j.jcrc.2016.07.015

3. Christian MD, Devereaux AV, Dichter JR, Rubinson L, Kissen N (2014) Introduction and executive summary: care of the critically ill and injured during pandemics and disasters: CHEST consensus statement. Chest 146:8S-34S. https://doi.org/10.1378/chest.14-0732

4. Einav S, Hick JL, Hanfling D et al (2014) Surge capacity logistics: Care of the critically ill and injured during pandemics and disasters: CHEST consensus statement. Chest 146:e17S-e43S. https://doi.org/10.1378/chest. $14-0734$ 